\title{
Why it is time to develop the use of cognitive event-related potentials in the treatment of psychiatric diseases
}

This article was published in the following Dove Press journal:

Neuropsychiatric Disease and Treatment

27 November 2013

Number of times this article has been viewed

\author{
Salvatore Campanella \\ Laboratory of Medical Psychology \\ and Addictology, ULB Neuroscience \\ Institute (UNI), Université Libre de \\ Bruxelles, Brussels, Belgium
}

Correspondence: Salvatore Campanella Laboratory of Psychological Medicine and Addictology (UNI-ULB), CHU Brugmann - Secretary of the Department of Psychiatry, 4 Place Vangehuchten, 1020 Brussels, Belgium

Tel +32 24773465

Fax +3224772162

Email salvatore.campanella@chubrugmann.be; salvatore.campanella@ulb. ac.be

\begin{abstract}
The relapse rate for many psychiatric disorders is staggeringly high, indicating that treatment methods combining psychotherapy with neuropharmacological interventions are not entirely effective. Therefore, in psychiatry, there is a current push to develop alternatives to psychotherapy and medication-based approaches. Cognitive deficits have gained considerable importance in the field as critical features of mental illness, and it is now believed that they might represent valid therapeutic targets. Indeed, an increase in cognitive skills has been shown to have a long-lasting, positive impact on the patients' quality of life and their clinical symptoms. We hereby present four principal arguments supporting the use of event-related potentials (ERP) that are derived from electroencephalography, which allow the identification of specific neurocognitive deficiencies in patients. These arguments could assist psychiatrists in the development of individualized, targeted therapy, as well as a follow-up and rehabilitation plan specific to each patient's deficit. Furthermore, they can be used as a tool to assess the possible benefits of combination therapy, consisting of medication, psychotherapy, and "ERP-oriented cognitive rehabilitation". Using this strategy, specific cognitive interventions could be planned based on each patient's needs, for an "individualized" or "personalized" therapy, which may have the potential to reduce relapse rates for many psychiatric disorders. The implementation of such a combined approach would require intense collaboration between psychiatry departments, clinical neurophysiology laboratories, and neuropsychological rehabilitation centers.
\end{abstract}

Keywords: neuropsychiatry, relapse, neurophysiology, neuropsychology, cognitive rehabilitation, event-related potentials

\section{Introduction}

According to the World Health Organization, “... there is no health without mental health...". ${ }^{1}$ Mental disorders make up an important independent contribution to the burden of non-communicable diseases worldwide. In fact, neuropsychiatric conditions account for up to one quarter of all disability-adjusted life years (ie, the sum of years lived with disability and years of life lost). ${ }^{1}$ A psychiatrist's main responsibility is to render accurate diagnoses that guide the selection of appropriate medication for severely mentally ill patients. ${ }^{2}$ A crucial problem in psychiatry, which affects both research and clinical work, is that in practice it is difficult to strictly apply the scientific method to the investigation of human mental illness, since the evaluations should (at least) include analyses of both the mind and the brain when assessing normal and aberrant behaviors. ${ }^{3}$ A consequence of this complexity is that, even if psychopathological states can effectively be alleviated by a combination of psychotherapy and neuropharmacological interventions, the relapse rate for several psychiatric disorders 
is tremendously high. ${ }^{4}$ For example, the relapse rate for manic episodes in bipolar disorders was found to be $50 \%$ at 1 year, and $70 \%$ at 5 years. ${ }^{5}$ Similarly, the relapse rate following a first episode of schizophrenia was reported to be $35 \%$ at 18 months, and $74 \%$ at 5 years. ${ }^{6}$ In other words, there is still much to learn in order to fill this existing gap between a "partial recovery", which allows patients to return home, and the achievement of a "complete recovery".

The complexity surrounding the treatment of mental illness is also illustrated by alcohol dependence, which constitutes one of the most severe and widespread public health problems. Indeed, it is estimated that $3 \%-8 \%$ of all deaths and $4 \%$ of all disability-adjusted life years worldwide are attributable to alcohol. ${ }^{1}$ Without intervention, detoxification alone does little to prevent an alcoholic's subsequent relapse. During clinical trials, placebo control groups showed a relapse rate of up to $85 \%$, even if hospitalized until complete remission of the physical withdrawal symptoms. ${ }^{8}$ Similarly, there is little evidence supporting that antidepressants alter in any way the risk factors that lead to relapse and recurrence. Consequently, most patients with chronic or recurrent depression are encouraged to stay on medication indefinitely. ${ }^{9}$ In addition, while it has been well established that maintenance treatment with antipsychotic medication decreases relapse rates, a substantial proportion of patients suffering from schizophrenia either relapse despite taking medication or have trouble adhering to antipsychotic treatment. ${ }^{10}$ All of the above fuels the current strong interest for the development of alternatives to psychotherapy and medication. ${ }^{11}$ In recent years, we have seen the emergence of several intervention strategies aimed at improving psychiatric treatment, such as multisystemic, ${ }^{12}$ cognitive behavioral, ${ }^{13}$ or mindfulness ${ }^{14}$ therapies. The goal of these "new" interventions is evidently not to discredit existing treatment methods, but to provide a complementary set of tools to be used by clinicians to improve current patient assessment.

Therefore, the purpose of this paper is to present four key arguments that support the use of event-related potential (ERP)-based cognitive assessment in combination with psychotherapy and medication to develop more efficient psychiatric therapies. It is now largely accepted that psychiatric disorders are brain disorders, ${ }^{15}$ and brain-related tools, such as electroencephalography $(\mathrm{EEG})^{16}$ or repetitive transcranial magnetic stimulation (rTMS), ${ }^{17}$ have already proven their value for the management of psychiatric diseases. For instance, the combined use of rTMS and EEG enables the noninvasive evaluation of functional connections between brain areas, and provides a tool to investigate cortical activity. ${ }^{18}$ Thus, a non-invasive input (TMS) known spatial and temporal characteristics with directional and precise chronometric data can be applied to the study of the brain's local reactivity and the interactions between different brain regions. ${ }^{19}$ This emerging technique allows for instance to observe that, during an 8 to 10 minute long EEG session with single-pulse TMS over the right premotor cortex, patients suffering from schizophrenia present reduced evoked gamma oscillations in the frontal cortex, reinforcing the hypothesis of an intrinsic dysfunction of the frontal thalamo-cortical circuit. ${ }^{20}$ In this paper, we focus on ERPs, since by allowing the evaluation of the entire information processing stream, they can help pinpoint the specific neurocognitive functions that should be rehabilitated in each patient through specific and individualized cognitive remediation procedures. The aim of this paper is not to provide an exhaustive review of the literature pertaining to ERPs and psychiatric disorders, but to systematically illustrate four reasons why we think that it is now time to incorporate cognitive ERPs as a requisite step in clinical evaluations and use them to direct the development of an individualized therapy for mentally ill patients. This approach should also include a cognitive neuropsychological rehabilitation step, as an integrative part of the "whole process" that is classically only based on psychotherapy and medication. We will discuss the advantages, limitations, and perspectives associated with the clinical use of ERPs and we will propose new ways in which this technique can be effectively incorporated into patient care.

\section{Argument I: the cognitive process is a key element of psychopathological states}

Cognitive deficits have gained considerable importance in the field as critical features of mental illness, and it is now believed that they might represent valid therapeutic targets. ${ }^{21}$ Indeed, several studies have provided consistent evidence that mental illness involves significant cognitive impairment. For instance, virtually all areas of cognition, such as perception, attention, and memory, are known to be impaired in the schizophrenic individual, ${ }^{22}$ as well as in patients of other psychiatric afflictions, such as euthymic bipolar disorder, ${ }^{23}$ alcohol-dependence, ${ }^{24}$ or attention-deficit/ hyperactivity disorder (ADHD). ${ }^{25}$ In schizophrenic patients, literature data reports a substantial decrease in cognitive function of about 1.0-1.75 standard deviations below the normal mean. ${ }^{26}$ Moreover, some variability in the cognitive domain 
was also reported in euthymic bipolar patients (large effect sizes $[\mathrm{d} \geq 0.8]$ for executive functions and small effect sizes $[0.2 \leq \mathrm{db} 0.5]$ for sustained attention). ${ }^{23}$ However, a proven connection between the onset of a mental illness and the presence of cognitive impairment would be needed in order to establish the requirement for cognitive therapeutics. ${ }^{27}$ Additionally, while there is overwhelming evidence that medication can reduce the severity of clinical symptoms, cognitive gains are either poor or undetectable. ${ }^{21}$ Thus, it is currently accepted that cognitive impairment and symptomatic manifestations of mental illness require separate therapeutic approaches. ${ }^{28}$

Cognitive retraining procedures (CRPs) have brought new applications to psychiatric rehabilitation. ${ }^{29}$ Cognitive remediation is a behavioral intervention designed to improve cognition in patients who have suffered a cognitive decline. ${ }^{30}$ The major elements of psychiatric rehabilitation focus on highlighting the patient's strengths, improving vocational/ social competencies, working with environmental facts of an individual's life, and instilling a sense of mastery and hope. ${ }^{31}$ From this perspective, cognitive retraining techniques range from assistive or prosthetic devices (including computers, diaries, and/or lists) to approaches that focus on enhancing the patient's efficiency, (eg, mnemonic strategies). ${ }^{32}$ Many studies have demonstrated that the use of CRPs can enhance cognitive performance in diverse psychiatric diseases, ${ }^{33}$ and that these improvements are stable up to at least 6 months after the treatment is discontinued. ${ }^{34}$ Additionally, CRPrelated cognitive gains have been proved to translate to improvements in real world activities, ${ }^{35}$ with greater impact than any drug treatment. ${ }^{21}$ For example, in a single case study involving a schizophrenic patient,${ }^{35}$ Levaux et al demonstrated that the usage of an attention training technique leads to the following beneficial effects: i) at the cognitive level, a specific improvement in selective attention, attention switching, and resistance to distractive interference; and ii) at the clinical level, a reduction in intrusive thoughts in daily life and a general decrease in symptoms stable up to at least 6 months. Overall, cognitive remediation is a therapy that engages patients in learning activities that enhance the neurocognitive skills relevant to their chosen recovery goals. ${ }^{36} \mathrm{~A}$ number of approaches to remediating cognition in schizophrenia have been developed and studied over the last 20 years. At the time of writing this article, this literature has been reviewed in six meta-analytic studies. Five of these, while differing in focus, determined that it has moderate to large effect sizes, ${ }^{37-41}$ the remaining one didn't. ${ }^{42}$
Moreover, with the spread of CRP's application to patients suffering from schizophrenia, positive outcomes with CRP for other psychiatric disorders, such as unipolar depression, ${ }^{43}$ social phobia, ${ }^{44}$ ADHD, ${ }^{45}$ and alcohol dependence ${ }^{46}$ have begun to appear in the literature. Overall, these data are consistent with the general idea that recognizing the nature of the cognitive impairment involved in a specific mental illness will be very useful for the development of treatment and rehabilitation strategies. ${ }^{29}$

Although the usefulness of CRPs for the treatment of psychiatric disorders is gradually becoming more accepted, several questions regarding how to use them in clinical practice still remain to be resolved. Among other concerns, it is unknown whether cognitive impairments will be detected in all patients or just on a case-by-case basis. ${ }^{27}$ Also, the assignment of impairment requires the comparison of patients' results to those of a "normal" or "standard" cognitive control; however, normal values may be difficult to define, mainly because of the healthy individuals' variability in cognitive function. In fact, it appears that, for instance, around $20 \%$ of schizophrenics generally perform within the normal range, only showing subtle evidence of impairment in specific cognitive domains. ${ }^{47}$ Indeed, the human mind can be defined by several cognitive domains, such as attention, memory, language, or executive functions, any of which may prove to be altered. ${ }^{48}$ This requires rehabilitation specialists (usually neuropsychologists) to thoroughly assess the cognitive status of each domain in order to decide the appropriate rehabilitative treatment. This process may be complicated by two factors: i) the fractionation of each cognitive domain implies the involvement of various neural networks (eg, executive function refers to a wide range of cognitive processes [including problem-solving, flexibility, inhibition, planning, and decision-making], and a patient's performance in one executive function may have little or no predictive value for how the patient will perform in another); ${ }^{49}$ and ii) time pressure is an important feature of the clinical examination, because cognitive screening needs to identify genuine impairment in as little time as possible, using an easily administered instrument. ${ }^{50}$

In the following arguments, we will describe how and why we believe that cognitive ERPs can aid in the cognitive screening process. A general screening of main cognitive functions can be achieved by performing rapid tasks, which are easily implementable in clinical practice and have the potential to index the cognitive areas that require further assessment and/or rehabilitation by neuropsychologists. 


\section{Argument 2: the ERP method, a highly adapted tool to investigate the sequence of cognitive processes}

The main goal of cognitive psychology has been to define the different stages that are required to achieve a final performance for each human cognitive function. ${ }^{51}$ Indeed, every cognitive function (eg, recognizing an emotion on a face, recalling an event, reading a text, or making a decision) is associated with various cognitive stages. In fact, advances in brain imaging techniques (namely positron emission tomography [PET] and functional resonance magnetic imaging [fMRI]) have revealed that each of the serial cognitive stages is implemented within separate neural processes in order to achieve normal function. ${ }^{52}$

The fact that healthy cognitive functions are implemented within distributed neural networks has important implications for the understanding of mental disorders. Firstly, the use of advanced imaging methods enabled the study of the neurobiology of mental disorders, confirming the fact that mental disorders have a neurobiological component (ie, they are not only functional or psychogenic conditions). ${ }^{53}$ Secondly, imaging technology contributed insight into the pathological mechanisms of mental disorders, demonstrating that a functional alteration may result from an isolated brain lesion (eg, after a stroke) or deficient interactions between different brain regions (functional connectivity) ${ }^{54}$ In this regard, imaging analysis can also provide a better understanding of the mechanisms of action of psychopharmaceuticals, potentially aiding in the search for novel therapeutic targets. ${ }^{53}$

The study of blood flow (fMRI) or glucose metabolism (PET) in certain areas of the brain during activation tasks enables the identification of the areas of the brain that are activated or deactivated during specific cognitive tasks. Therefore, by comparing patients to controls, we can observe which brain activities are deficient. However, from a purely cognitive perspective, these data have an important limitation, residing on the fact that brain images are averaged on seconds. Thus, it is impossible to relate specific brain activations to the different cognitive stages involved in the task. ${ }^{55}$ In other words, the excellent anatomical resolution of brain imaging techniques (3-4 mm for fMRI) might allow the visualization of the brain network involved in a certain task, but its coarse temporal resolution (1-2 seconds) impedes the determination of the activation sequence. For this purpose, we should recur to electrophysiological tools, and more precisely, to cognitive ERPs. ${ }^{56}$

ERPs, which are derived from electroencephalography, are highly sensitive and have the potential to monitor brain electrical activity with a fine temporal resolution (on the order of milliseconds). Thus, when healthy individuals perform a cognitive task, it is possible to observe the various electrophysiological components representing the cognitive stages utilized to achieve "normal" performance. ${ }^{56}$ Figure 1 illustrates the different electrophysiological components representing the various cognitive stages needed to perform a simple task.

Another highly valuable aspect of cognitive ERPs is that they can permit the identification/detection of the electrophysiological component(s) consistent with the onset of a dysfunction, allowing the inference of impaired cognitive stages. ${ }^{56}$ In other words, by using a well characterized task and analyzing which ERPs show decreased amplitude and/or delayed latency compared to normal values, it is possible to deduce which ERP component is responsible for the cognitive deficit. ${ }^{56}$ Figure 2 illustrates the well-known P300 component, a late, long-lasting parietal positive wave, which is decreased and delayed in alcoholic patients compared to matched controls. However, while P300 deficit is common in many psychiatric afflictions, there is also an abundance of literature reporting; for instance, many earlier ERP components, such as the mismatch negativity or the sensory gating $\mathrm{P} 50$, but also later ones, such as the N400 indexing language semantic processes, are affected in alcoholic, ${ }^{57}$ as well as in schizophrenic $^{58}$ patients. This suggests that during illness, various cognitive stages of the information-processing stream may be affected, and that the recorded ERP components may be considered as biological markers of the disease, indexing specific pathophysiological mechanisms that may or may not recover with the illness remission. ${ }^{59}$

Obviously, ERPs also suffer from some limitations. Their spatial resolution is very weak, so that inferences can only be made on the neural generators of the observed components, and parameters of ERPs (latency and amplitude) are subject to large inter-individual differences, so that the collection of normative data may prove fastidious. ${ }^{56}$ Nevertheless, cognitive ERPs are advantageous, because they allow the assessment of the different stages used in the informationprocessing stream during performance of a task. Therefore, how and why could this technique be useful to psychiatrists in their daily clinical practice?

\section{Argument 3: a similar behavioral deficit may originate from different cognitive levels}

The most widely used and studied ERP component is the P300 component. ${ }^{60}$ This component is of particular interest 


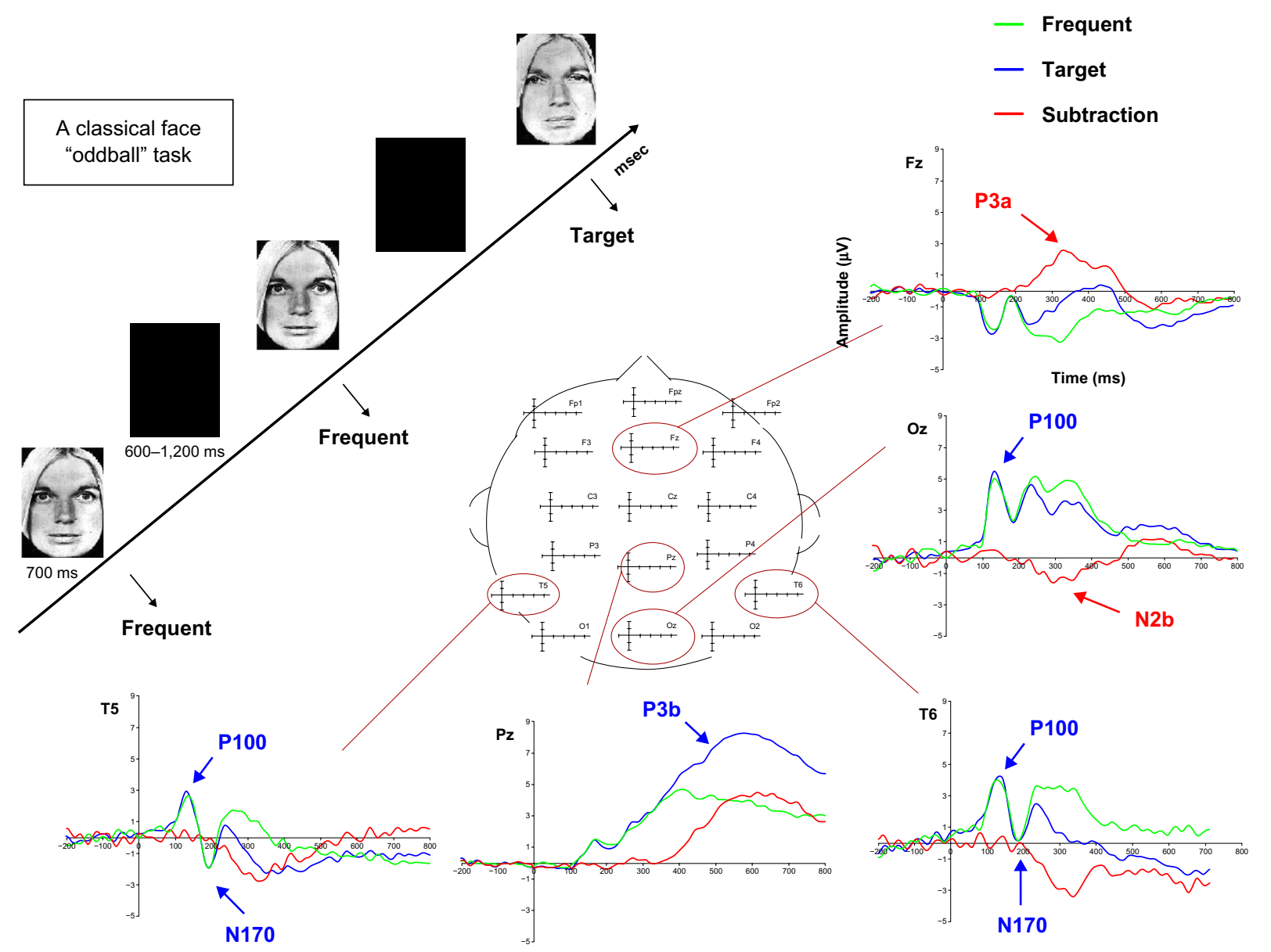

Figure I Illustration of the different ERP components recorded in a face-oddball task, in which participants have to detect as quickly as possible the appearance of a target "emotional sad" face among a train of frequent neutral faces by clicking on a button. The PI00 component, recorded around I00 ms, refers to the visual perceptive analysis of the stimulus, and can be modulated through attention by different mechanisms such as complexity or motivation. The NI70 component is a bilateral occipito-temporal negativity, recorded around $170 \mathrm{~ms}$ that refers to the structural encoding of facial information in order to generate a representation of the observed face in short-term memory. The N2b/P3a is a bipolar complex, obtained by subtracting the activity recorded for frequent stimuli from the one obtained for targets, with a posterior negativity recorded around $250 \mathrm{~ms}$, referring to the allocation of attentional resources, while the frontal P3a is more sensitive to stimulus novelty. This complex is functionally seen as the switch of attention needed to process something new appearing in the environment. Finally, the P3b component is a parietal activity indexing pre-motor response stages that shows that the facial representation created in short-term memory for frequent faces has been updated, so that a behavioral motor response may be prepared.

Note: Adapted with permission from Maurage et al94 @ 2009 Canadian Medical Association and Maurage et al. Electrophysiological correlates of the disrupted processing of anger in alcoholism. Int J Psychophysiol. 2008:70(I):50-62. ${ }^{95}$ Copyright (C) 2008 Elsevier Inc.

Abbreviation: ERP, event-related potentials.

because it is functionally related to various complex cognitive processes, such as decision-making and cognitive closure phenomena, which are associated with different types of response-related cognitive activities. ${ }^{61}$ Many studies investigating P300 in dementia, schizophrenia, depression, alcoholism, drug addiction, anxiety, or personality disorders have described disturbances in recorded latency and amplitude values of this component compared to healthy individuals. These differences have been associated with deficits in behavioral performance and can indicate the severity of a clinical state, as well as its possible evolution. ${ }^{62}$

For example, if we consider the literature on the ability to recognize emotional facial expressions (EFE) in psychiatric populations, two main points emerge: i) at a behavioral level, many patients with psychiatric diseases display a deficit in emotional recognition, which can be indexed by decreased performance rates and longer response latencies compared to controls; ${ }^{63}$ and ii) at an electrophysiological level, this behavioral deficit is associated with a decreased and delayed P300 component. ${ }^{64}$ However, an important question in the field has revolved around the events occurring in the information-processing stream prior to the $\mathrm{P} 300$ component (ie, before the response-related stages). By using an emotional oddball task, ${ }^{64,65}$ we demonstrated that even if different psychopathological populations displayed a similar behavioral deficit in EFE recognition, this deficit originated at different cognitive levels, as indexed by cognitive ERPs. Indeed, while patients suffering from alcohol dependence or schizophrenia displayed altered ERP components affecting all informationprocessing streams (from perceptive-visual P100, to attention 


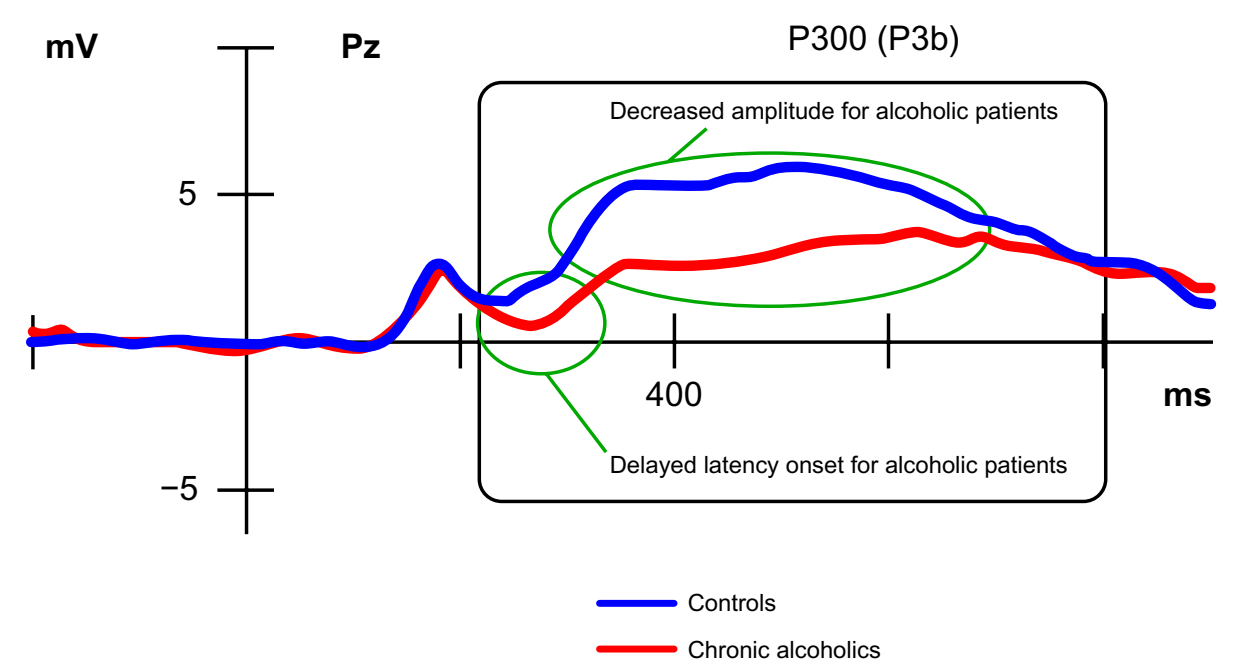

Figure 2 Illustration of a disturbed P300 (P3b) component resulting from the comparison of alcoholic patients with healthy matched controls. Note: Adapted with permission from Maurage et al ${ }^{94}$ (C) 2009 Canadian Medical Association.

N2b and response-related P300 components), ${ }^{66,67}$ depressed people exhibited only a disturbed P300 component (with intact earlier components). ${ }^{6}$ On the other hand, ecstasy users presented differences starting at the level of attention (N2b). ${ }^{69}$ In other words, similar patterns at the behavioral level can result from different disturbances in cognitive processes from one population to another. This point has great clinical relevance, because it suggests that a similar pattern of deficit may be attributable to different neurocognitive disturbances, supporting the notion that similar behavioral deficits should be differently rehabilitated.

From a clinical point-of-view, it might not be a surprise that there are neurocognitive differences linked to different neuropsychiatric afflictions, especially considering the specificity of the pathophysiological mechanisms associated with distinct mental illnesses. However, interestingly, we also observe that such neurocognitive differences can be seen between individuals within a specific population that have a certain type of behavioral deficit. For instance, among anxious patients, Rossignol et al showed that those suffering from generalized anxiety disorder only displayed P300 differences when confronted with emotional oddball task, ${ }^{70}$ whereas people with social anxiety showed earlier differences starting from the perceptive P100 component. ${ }^{71}$ This suggests that social anxiety and trait anxiety should be considered separately and treated independently. ${ }^{72}$ This realization is of great relevance, because it suggests that patients who display a similar group of symptoms and belong to a closed psychiatric category might have deficits that originate from different levels of the cognitive process.

Overall, the monitoring of ERPs is useful for identifying the cognitive origin of a behavioral deficit. By temporally analyzing the information-processing stream, the root causes of behavioral problems can be identified as perceptive, attention, or response related. Current data suggest that such differences not only exist between the various neuropsychiatric afflictions, but also among different patients categorized in the same psychiatric category. Therefore, the next important question is: how can such information be useful for psychiatrists?

\section{Argument 4: once specific cognitive deficiencies are defined, directed cognitive retraining procedures may help to alleviate symptoms in homogeneous subgroups of patients}

Thanks to an optimal temporal resolution, ERPs allow the assignment of a behavioral deficit to its cognitive origin. This feature of ERP analysis is of great clinical relevance, since it suggests that each patient's behavioral deficit can be specifically treated. However, even if the importance of indexing cognitive disturbances is well-established in psychiatric clinical settings, the manner with which to efficiently perform cognitive analyses is a matter of debate. This is mainly due to cognition fractionation, which means that a global assessment of all cognitive functions may take several hours to perform, and this is incompatible with both patients' statuses and consultation pressures. Nevertheless, ERP analysis is non-invasive, painless, and cheap. In addition, ERPs provide the most informative profile for psychiatrists regarding the cognitive deficits presented by their patients, especially when examining ERP components that are wellcharacterized in terms of eliciting stimuli, technical recording 
methods, and quantification. These defined ERPs are easily implementable in clinical practice and are operationally related to the neurocognitive processes they reflect. ${ }^{73}$

We would especially like to emphasize that the most useful components span different stages of the information processing stream, including P50, mismatch negativity (MMN), P300, NoGo P300, and error-related negativity (ERN). The P50 component is the earliest to be observed (around $50 \mathrm{~ms}$ ) and the smallest in amplitude of the auditory ERPs. The P50 sensory gating effect refers to an amplitude reduction in P50 upon exposure to a second stimulus in a pair of identical stimuli presented with a short inter-stimulus interval. P50 gating is one of the earliest, measurable brain sensory processing steps that are linked to screening-out and attention filtering mechanisms of redundant incoming information. ${ }^{74}$ MMN (also called N2a) is an ERP component with peak latency around $150 \mathrm{~ms}$ after stimulus and maximal amplitude at the frontal scalp locations. MMN is usually evoked by a physically deviant auditory stimulus that occurs in a series of frequent standard stimuli. It is believed to reflect cortical information processing at the earliest level of the sensory cortex; however, recent findings suggest that the transient auditory sensory memory representation underlying the MMN is facilitated by a long-term memory representation of the corresponding stimulus. ${ }^{75}$ The $\mathrm{P} 300$ component (or P3) is a long-lasting positive component that occurs between 300 and $700 \mathrm{~ms}$ after stimulation. P3 is thought to reflect premotor processes, such as short-term memory updating or cognitive closure mechanisms. ${ }^{58}$ The NoGo P300 has been identified as one of the markers for response inhibition, ${ }^{76}$ which involves the activation of the executive system of the frontal lobes. ${ }^{77}$ The neural basis for this executive system is believed to be a distributed circuitry, which involves the following: the prefrontal areas and anterior cingulated gyrus; ${ }^{78}$ the orbitofrontal cortex $;{ }^{79}$ the ventral frontal regions $;{ }^{80}$ the parietal, dorsal, and ventral prefrontal regions $;{ }^{81}$ and the premotor and supplementary motor areas. Finally, ERN represents a neural measure of error processing that peaks $50 \mathrm{~ms}$ after subjects make mistakes. This component is linked to the subject's ability to evaluate his actions as "worse than expected", and constitutes a "learning signal" that can subsequently be used to adjust behavior. ${ }^{82}$

It has been suggested that the analysis of a weighted combination of these electrophysiological features may provide greater diagnostic power than any single endophenotype alone. ${ }^{83}$ For instance, Price et $\mathrm{al}^{84}$ compared and contrasted four electrophysiological components, analyzing their covariance on a single cohort of schizophrenic patients, family members, and controls. Their findings revealed that the use of the electrophysiological battery provided novel information on the characteristics of the different groups. In particular, they highlighted the heterogeneity of electrophysiological features among the groups and demonstrated that the combined analysis could serve to minimize the impact of such heterogeneity. ${ }^{84}$

We propose the use of P50, MMN, P300, NoGo P300, and ERN to facilitate the indexing of cognitive stages affected in psychiatric patients by assessing, respectively, the attentionfiltering, early perceptive discriminative, response-related decisional, inhibitory, and self-action evaluation processes. Indeed, neurocognitive processes (indexing attention, executive functions, and meta-cognition) have been linked to psychopathology. ${ }^{85}$ Once cognitive disturbances are characterized through ERP screening for individual patients, psychiatrists will be able to orient the "cognitive" treatment (individually or in groups that present homogeneous patterns of cognitive deficits). More precisely, specific cognitive retraining procedures could be used to target deficits and increase cognitive efficiency, which has already been shown to reduce clinical symptoms. ${ }^{86,87}$ In this regard, there are currently many CRPs available, including those directed at attention mechanisms (increasing or decreasing the attention resources devoted to a specific cue), ${ }^{88}$ for which compelling evidence has already been gathered in anxiety disorders. Indeed, behavioral and ERP studies have shown that attention training can alter threat bias, influence vulnerability to stress, and reduce clinical anxiety symptoms by modulating top-down processes of attention control rather than processes of early attention orienting. ${ }^{89}$ In the same vein, there are also available CRPs increasing organization, goal achievement, ${ }^{90}$ cognitive control (ie, inhibitory skills), ${ }^{46}$ and even self-monitoring. ${ }^{91}$

\section{Conclusion}

\section{Creating a multi-disciplinary approach to mental illness, including neurophysiologists and neuropsychologists}

Mental illness is commonly managed with a combined approach involving psychotherapy and pharmacological intervention. This strategy is certainly useful for some patients, but the relapse rate remains tremendously high for several psychiatric disorders. Thus, in psychiatry there is currently a recognized need for alternatives to psychotherapy and medication. ${ }^{11}$ Cognitive deficits have gained considerable importance in the field as critical features of mental illness. ${ }^{21}$ 
Therefore, in the present paper, we suggest that a possible way to increase the efficiency of psychiatric treatment is to include ERPs and CRPs in clinical practice, combining these techniques with psychotherapy and medication. However, this would require a significant collaboration between psychiatry departments, clinical neurophysiology laboratories, and neuropsychological rehabilitation centers. In this view, a psychiatrist may prescribe a "complete ERP screening" to his/her patients, which is easy to perform and will not be time-consuming if only the main cognitive domains are analyzed (attention, execution, and self-monitoring). The basic purpose of these ERP screening tests will be to indicate the likelihood of genuine cognitive impairment, which can be inferred through the comparison of the patient's results with reference controls. Thus, a borderline score or a very impaired score (along with supporting history) might lead the psychiatrist to order a more specialized assessment of cognition (eg, by neuropsychologists), and thus thoroughly identify which cognitive domain(s) to rehabilitate. Indeed, CRPs have been proven to have a positive impact on daily quality of life ${ }^{92}$ and on clinical symptoms. ${ }^{86,87}$ Hence, psychiatric treatment data from neurophysiologists (ERPs) could help neuropsychologists decide which cognitive processes to rehabilitate through CRPs in order to enhance the daily quality of life and soften the clinical symptoms of individual patients. Ultimately, based on the fact that the long-lasting, positive effects of CRPs on clinical symptoms have already been demonstrated, ${ }^{35,87}$ we are convinced that the combination of this cognitive procedure (involving ERPs and CRPs) with psychotherapy and medication could lead to decreased relapse rates.

\section{There is still a long way to go before this strategy can be implemented into daily clinical practice}

Although the rationale of this proposal is highly reliable and theoretically grounded, we are entirely aware that it is currently impossible to implement this proposal in clinical practice. Indeed, the principal concern relates to the ERP screening procedure. To date, the majority of ERP related studies comparing psychiatric populations to matched controls used grand-averaged data (ie, mean data comparing a group of healthy individuals to a group of patients). However, in daily clinical practice, in order to be able to decide whether an individual patient presents a genuine cognitive impairment, we need to develop normal and impaired ranges for ERP parameters (amplitude and/or latency). In other words, it is necessary to accumulate unambiguous referenced normative data in order to determine whether a patient requires cognitive rehabilitation. In this regard, there already exist guidelines for some ERP components (MMN and P300), which could be clinically implemented. ${ }^{93}$ For other components, normative data remains to be gathered (P50, NoGo P300, and ERN). Therefore, there is an urgent need for further research to develop multisite guidelines that can be compared and used across studies by recording a battery of these five electrophysiological measures (P50, MMN, P300, NoGo P300, and ERN).

We propose that the time needed for ERP screening could be greatly reduced by focusing on the five ERP components described above, while other components (eg, contingent negative variation, P100, N300, and N400) and other related cognitive processes (eg, anticipation effects, visual, emotional, or language processes) may be of great interest. Here, we have focused on the components related to attention (through MMN, P50), execution (NoGo P300), memory and decision-making capacity (P300), and self-monitoring (ERN), which are the ones that have been primarily screened in psychopathological states. ${ }^{85}$ Nevertheless, if our approach proves to be efficient, other batteries using different components could also be developed for future clinical use.

To conclude, there is a general agreement that a multidisciplinary approach is required for the successful treatment of mental illness. In this proposal, we have suggested that psychiatrists should continue to maintain their classic collaboration with psychologists, who are trained to provide psychotherapy. However, we now suggest that neurophysiologists and neuropsychologists may be crucial to aid psychiatrists in the identification of cognitive processes that should be rehabilitated on a patient-by-patient basis. The resulting combined approach (ie, medication, psychotherapy, and "ERP-oriented cognitive rehabilitation") should reduce relapse rates for many psychiatric afflictions, because individual cognitive interventions would be specifically targeted based on each patient's needs, thus providing an "individualized" or "personalized" medicine. Therefore, the challenge of future studies will be to highlight whether this procedure is efficient enough to be incorporated into a psychiatrist's current treatment method toolbox.

\section{Acknowledgments}

Salvatore Campanella is a Research Associate at the Belgian Fund for Scientific Research (FNRS, Belgium). 


\section{Disclosure}

This research was funded by the Belgian Fund for Scientific Research (FNRS, Belgium), but this did not exert any editorial direction or censorship on any part of this article. The author reports no other conflicts of interest in this work.

\section{References}

1. Prince M, Patel V, Saxena S, et al. No health without mental health. Lancet. 2007;370(9590):859-877.

2. Basco M, Bostic J, Davies D, et al. Methods to Improve Diagnostic Accuracy in a Community Mental Health Setting. Am J Psychiatry. 2000;157:1599-1605.

3. Epstein J, Stern E, Silbersweig D. Neuropsychiatry at the millenium: the potential for mind/brain integration through emerging interdisciplinary research strategies. Clin Neurosci Res. 2001;1:10-18.

4. Kohn R, Saxena S, Levav I, Saraceno B. The treatment gap in mental health care. Bull World Health Organ. 2004;82(11):858-866.

5. Perry A, Tarrier N, Morriss R, McCarthy E, Limb K. Randomised controlled trial of efficacy of teaching patients with bipolar disorder to identify early symptoms of relapse and obtain treatment. BMJ. 1999; 318(7177):149-153.

6. Robinson D, Woerner MG, Alvir JM, et al. Predictors of relapse following response from a first episode of schizophrenia or schizoaffective disorder. Arch Gen Psychiatry. 1999;56(3):241-247.

7. Andreasen NC, Carpenter WT, Kane JM, Lasser RA, Marder SR, Weinberger DR. Remission in schizophrenia: proposed criteria and rationale for consensus. Am J Psychiatry. 2005;162(3):441-449.

8. Boothby LA, Doering PL. Acamprosate for the treatment of alcohol dependence. Clin Ther. 2005;27(6):695-714.

9. Hollon SD, Thase ME, Markowitz JC. Treatment and prevention of depression. Psychol Science. 2002;3:1-39.

10. Leucht S, Barnes TR, Kissling W, Engel RR, Correll C, Kane JM. Relapse prevention in schizophrenia with new-generation antipsychotics: a systematic review and exploratory meta-analysis of randomized, controlled trials. Am J Psychiatry. 2003;160(7):1209-1222.

11. Dobson KS, Hollon SD, Dimidjian S, et al. Randomized trial of behavioral activation, cognitive therapy, and antidepressant medication in the prevention of relapse and recurrence in major depression. J Consult Clin Psychol. 2008;76(3):468-477.

12. Henggeler SW. Multisystemic Therapy: An overview of clinical procedures, outcomes, and policy implications. Child Psychol Psychiatry Rev. 1999;4(1):1-10.

13. Pike KM, Walsh BT, Vitousek K, Wilson GT, Bauer J. Cognitive behavior therapy in the posthospitalization treatment of anorexia nervosa. $\mathrm{Am}$ J Psychiatry. 2003;160(11):2046-2049.

14. Chiesa A, Serretti A. Mindfulness based cognitive therapy for psychiatric disorders: a systematic review and meta-analysis. Psychiatry Res. 2011;187(3):441-453.

15. Leshner AI. Addiction is a brain disease, and it matters. Science. 1997;278(5335):45-47.

16. Pogarell O, Hegerl U, Boutros N. Clinical neurophysiology services in psychiatry departments. Psychiatr Serv. 2005;56(7):871.

17. Slotema CW, Blom JD, Hoek HW, Sommer IE. Should we expand the toolbox of psychiatric treatment methods to include Repetitive Transcranial Magnetic Stimulation (rTMS)? A meta-analysis of the efficacy of rTMS in psychiatric disorders. J Clin Psychiatry. 2010;71(7): 873-884.

18. Lioumis P, Kicić D, Savolainen P, Mäkelä JP, Kähkönen S Reproducibility of TMS-Evoked EEG responses. Hum Brain Mapp. 2009;30(4):1387-1396.

19. McClintock SM, Freitas C, Oberman L, Lisanby SH, Pascual-Leone A. Transcranial magnetic stimulation: a neuroscientific probe of cortical function in schizophrenia. Biol Psychiatry. 2011;70(1):19-27.
20. Ferrarelli F, Massimini M, Peterson MJ, et al. Reduced evoked gamma oscillations in the frontal cortex in schizophrenia patients: a TMS/EEG study. Am J Psychiatry. 2008;165(8):996-1005.

21. Larøi F, Raballo A. Notes from Underground: Are Cognitive-Enhancing Drugs Respecting their Promises? Front Psychol. 2010;1:158.

22. Heinrichs RW, Zakzanis KK. Neurocognitive deficit in schizophrenia: a quantitative review of the evidence. Neuropsychology. 1998;12(3): 426-445.

23. Robinson LJ, Thompson JM, Gallagher P, et al. A meta-analysis of cognitive deficits in euthymic patients with bipolar disorder. $J$ Affect Disord. 2006;93(1-3):105-115.

24. Loeber S, Vollstädt-Klein S, von der Goltz C, Flor H, Mann K, Kiefer F. Attentional bias in alcohol-dependent patients: the role of chronicity and executive functioning. Addict Biol. 2009;14(2):194-203.

25. Castellanos FX, Sonuga-Barke EJ, Milham MP, Tannock R. Characterizing cognition in ADHD: beyond executive dysfunction. Trends Cogn Sci. 2006;10(3):117-123.

26. Heaton RK, Gladsjo JA, Palmer BW, Kuck J, Marcotte TD, Jeste DV. Stability and course of neuropsychological deficits in schizophrenia. Arch Gen Psychiatry. 2001;58(1):24-32.

27. Gold JM. Cognitive deficits as treatment targets in schizophrenia. Schizophr Res. 2004;72(1):21-28.

28. Bark N, Revheim N, Huq F, Khalderov V, Ganz ZW, Medalia A. The impact of cognitive remediation on psychiatric symptoms of schizophrenia. Schizophr Res. 2003;63(3):229-235.

29. Spaulding WD, Fleming SK, Reed D, Sullivan M, Storzbach D, Lam M. Cognitive functioning in schizophrenia: implications for psychiatric rehabilitation. Schizophr Bull. 1999;25(2):275-289.

30. Medalia A, Richardson R. What predicts a good response to cognitive remediation interventions? Schizophr Bull. 2005;31(4): $942-953$.

31. Lamb HR. A century and a half of psychiatric rehabilitation in the United States. New Dir Ment Health Serv. 2001:99-110.

32. Allen DN, Goldstein G, Seaton BE. Cognitive rehabilitation of chronic alcohol abusers. Neuropsychol Rev. 1997;7(1):21-39.

33. Hogarty GE, Flesher S, Ulrich R, et al. Cognitive enhancement therapy for schizophrenia: effects of a 2-year randomized trial on cognition and behavior. Arch Gen Psychiatry. 2004;61(9):866-876.

34. Wykes T, Reeder C, Williams C, Corner J, Rice C, Everitt B. Are the effects of cognitive remediation therapy (CRT) durable? Results from an exploratory trial in schizophrenia. Schizophr Res. 2003;61(2-3): $163-174$.

35. Levaux MN, Laroi F, Offerlin-Meyer I, Danion JM, Van der Linden M. The Effectiveness of the Attention Training Technique in Reducing Intrusive Thoughts in Schizophrenia: A Case Study. Clin Case Stud. 2011;10(6):466-484.

36. Medalia A, Choi J. Cognitive remediation in schizophrenia. Neuropsychol Rev. 2009;19(3):353-364.

37. Kurtz MM, Moberg PJ, Gur RC, Gur RE. Approaches to cognitive remediation of neuropsychological deficits in schizophrenia: a review and meta-analysis. Neuropsychol Rev. 2001;11(4):197-210.

38. Suslow T, Schonauer K, Arolt V. Attention training in the cognitive rehabilitation of schizophrenic patients: a review of efficacy studies. Acta Psychiatr Scand. 2001;103(1):15-23.

39. Krabbendam L, Aleman A. Cognitive rehabilitation in schizophrenia: a quantitative analysis of controlled studies. Psychopharmacology (Berl). 2003;169(3-4):376-382.

40. Twamley EW, Jeste DV, Bellack AS. A review of cognitive training in schizophrenia. Schizophr Bull. 2003;29(2):359-382.

41. McGurk SR, Twamley EW, Sitzer DI, McHugo GJ, Mueser KT. A metaanalysis of cognitive remediation in schizophrenia. Am J Psychiatry. 2007;164(12):1791-1802.

42. Pilling S, Bebbington P, Kuipers E, et al. Psychological treatments in schizophrenia: II. Meta-analyses of randomized controlled trials of social skills training and cognitive remediation. Psychol Med. 2002; 32(5):783-791. 
43. Elgamal S, McKinnon MC, Ramakrishnan K, Joffe RT, MacQueen G. Successful computer-assisted cognitive remediation therapy in patients with unipolar depression: a proof of principle study. Psychol Med. 2007;37(9):1229-1238.

44. Hofmann SG. Cognitive mediation of treatment change in social phobia. J Consult Clin Psychol. 2004;72(3):393-399.

45. Virta M, Vedenpää A, Grönroos N, et al. Adults with ADHD benefit from cognitive-behaviorally oriented group rehabilitation: a study of 29 participants. J Atten Disord. 2008;12(3):218-226.

46. Wiers RW, Eberl C, Rinck M, Becker ES, Lindenmeyer J. Retraining automatic action tendencies changes alcoholic patients' approach bias for alcohol and improves treatment outcome. Psychol Sci. 2011;22(4):490-497.

47. Kremen WS, Seidman LJ, Faraone SV, Toomey R, Tsuang MT. The paradox of normal neuropsychological function in schizophrenia. J Abnorm Psychol. 2000;109(4):743-752.

48. Ruff RM. A friendly critique of neuropsychology: facing the challenges of our future. Arch Clin Neuropsychol. 2003;18(8):847-864.

49. Chan RC, Shum D, Toulopoulou T, Chen EY. Assessment of executive functions: review of instruments and identification of critical issues. Arch Clin Neuropsychol. 2008;23(2):201-216.

50. Cullen B, O'Neill B, Evans JJ, Coen RF, Lawlor BA. A review of screening tests for cognitive impairment. J Neurol Neurosurg Psychiatry. 2007;78(8):790-799.

51. Bruce V, Young A. Understanding face recognition. Br J Psychol. 1986;77(Pt 3):305-327.

52. Haxby JV, Hoffman EA, Gobbini MI. The distributed human neural system for face perception. Trends Cogn Sci. 2000;4(6): 223-233.

53. Kasparek T. How and why psychiatrists should use imaging methods. Activ Nerv Super. 2010;52(3-4):118-127.

54. Greicius M. Resting-state functional connectivity in neuropsychiatric disorders. Curr Opin Neurol. 2008;21(4):424-430.

55. Calhoun VD, Adali T, Pearlson GD, Kiehl KA. Neuronal chronometry of target detection: fusion of hemodynamic and event-related potential data. Neuroimage. 2006;30(2):544-553.

56. Rugg MD, Coles MGH. Electrophysiology of Mind. Event-Related Brain Potentials and Cognition. Oxford: Oxford University Press, Oxford Psychology Series; 1995.

57. Campanella S, Petit G, Maurage P, Kornreich C, Verbanck P, Noël X. Chronic alcoholism: insights from neurophysiology. Neurophysiol Clin. 2009;39(4-5):191-207.

58. Campanella S, Guerit JM. How clinical neurophysiology may contribute to the understanding of a psychiatric disease such as schizophrenia. Neurophysiol Clin. 2009;39(1):31-39.

59. Campanella S, Pogarell O, Boutros N. Event-related potentials in substance use disorders: A narrative review based on papers from 1984 to 2012. Clin EEG Neurosci. Available from: http://eeg.sagepub. com/content/early/2013/10/07/1550059413495533.abstract. Accessed November 6, 2013.

60. Sutton S, Tueting P, Zubin J, John ER. Information delivery and the sensory evoked potential. Science. 1967;155(3768):1436-1439.

61. Polich J. Updating P300: an integrative theory of P3a and P3b. Clin Neurophysiol. 2007;118(10):2128-2148.

62. Hansenne M. Event-related brain potentials in psychopathology: clinical and cognitive perspectives. Psychologica Belgica. 2006;46(1-2): 5-36.

63. Power M, Dalgleish T. Cognition and Emotion: From Order to Disorder. Hove: Psychology Press; 1997.

64. Campanella S, Gaspard C, Debatisse D, Bruyer R, Crommelinck M, Guerit JM. Discrimination of emotional facial expressions in a visual oddball task: an ERP study. Biol Psychol. 2002;59(3):171-186.

65. Campanella S, Philippot P. Insights from ERPs into emotional disorders: an affective neuroscience perspective. Psychologica Belgica. 2006;46(1-2):37-53.
66. Campanella S, Montedoro C, Streel E, Verbanck P, Rosier V. Early visual components (P100, N170) are disrupted in chronic schizophrenic patients: an event-related potentials study. Neurophysiol Clin. 2006;36(2):71-78.

67. Maurage P, Philippot P, Verbanck P, et al. Is the P300 deficit in alcoholism associated with early visual impairments (P100, N170)? An oddball paradigm. Clin Neurophysiol. 2007;118(3):633-644.

68. Maurage P, Campanella S, Philippot P, et al. Alcoholism leads to early perceptive alterations, independently of comorbid depressed state: an ERP study. Neurophysiol Clin. 2008;38(2):83-97.

69. Mejias S, Rossignol M, Debatisse D, et al. Event-related potentials (ERPs) in ecstasy (MDMA) users during a visual oddball task. Biol Psychol. 2005;69(3):333-352.

70. Rossignol M, Philippot P, Douilliez C, Crommelinck M, Campanella S. The perception of fearful and happy facial expression is modulated by anxiety: an event-related potential study. Neurosci Lett. 2005;377(2): 115-120.

71. Rossignol M, Campanella S, Maurage P, Heeren A, Falbo L, Philippot P. Enhanced perceptual responses during visual processing of facial stimuli in young socially anxious individuals. Neurosci Lett. 2012;526(1):68-73.

72. Rossignol M, Campanella S, Bissot C, Philippot P. Fear of negative evaluation and attentional bias for facial expressions: an event-related study. Brain Cogn. 2013;82(3):344-352.

73. Pfefferbaum A, Roth WT, Ford JM. Event-related potentials in the study of psychiatric disorders. Arch Gen Psychiatry. 1995;52(7):559-563.

74. Adler LE, Pachtman E, Franks RD, Pecevich M, Waldo MC, Freedman R. Neurophysiological evidence for a defect in neuronal mechanisms involved in sensory gating in schizophrenia. Biol Psychiatry. 1982;17(6):639-654.

75. Näätänen R, Kujala $T$, Escera $C$, et al. The mismatch negativity $(\mathrm{MMN})$ - a unique window to disturbed central auditory processing in ageing and different clinical conditions. Clin Neurophysiol. 2012;123(3):424-458.

76. Smith JL, Johnstone SJ, Barry RJ. Effects of pre-stimulus processing on subsequent events in a warned $\mathrm{Go} / \mathrm{NoGo}$ paradigm: response preparation, execution and inhibition. Int J Psychophysiol. 2006;61(2): 121-133.

77. Kaiser S, Unger J, Kiefer M, Markela J, Mundt C, Weisbrod M. Executive control deficit in depression: event-related potentials in a Go/Nogo task. Psychiatry Res. 2003;122(3):169-184.

78. Posner MI, DiGirolamo GJ. Executive attention: conflict, target detection and cognitive control. In: Parasuraman $\mathrm{R}$, editor. The Attentive Brain. Cambridge: MIT Press; 1998:401-423.

79. Fuster JM. The Prefrontal Cortex: Anatomy, Physiology and Neuropsychology of the Frontal Lobe. 2nd ed. New York: Raven Press; 1989.

80. Brown GG, Kindermann SS, Siegle GJ, Granholm E, Wong EC, Buxton RB. Brain activation and pupil response during covert performance of the Stroop Color Word task. J Int Neuropsychol Soc. 1999;5(4):308-319.

81. Watanabe J, Sugiura M, Sato K, et al. The human prefrontal and parietal association cortices are involved in NO-GO performances: an eventrelated fMRI study. Neuroimage. 2002;17(3):1207-1216.

82. Holroyd CB, Coles MG. The neural basis of human error processing: reinforcement learning, dopamine, and the error-related negativity. Psychol Rev. 2002;109(4):679-709.

83. Calkins ME, Iacono WG. Eye movement dysfunction in schizophrenia: a heritable characteristic for enhancing phenotype definition. Am J Med Genet. 2000;97(1):72-76.

84. Price GW, Michie PT, Johnston J, et al. A multivariate electrophysiological endophenotype, from a unitary cohort, shows greater research utility than any single feature in the Western Australian family study of schizophrenia. Biol Psychiatry. 2006;60(1):1-10.

85. Green MF, Nuechterlein KH, Gold JM, et al. Approaching a consensus cognitive battery for clinical trials in schizophrenia: the NIMH-MATRICS conference to select cognitive domains and test criteria. Biol Psychiatry. 2004;56(5):301-307. 
86. Lecardeur L, Stip E, Giguere M, Blouin G, Rodriguez JP, ChampagneLavau M. Effects of cognitive remediation therapies on psychotic symptoms and cognitive complaints in patients with schizophrenia and related disorders: a randomized study. Schizophr Res. 2009;111(1-3): 153-158.

87. Park S, Püschel J, Sauter BH, Rentsch M, Hell D. Spatial working memory deficits and clinical symptoms in schizophrenia: a 4-month follow-up study. Biol Psychiatry. 1999;46(3):392-400.

88. Amir N, Weber G, Beard C, Bomyea J, Taylor CT. The effect of a single-session attention modification program on response to a publicspeaking challenge in socially anxious individuals. J Abnorm Psychol. 2008;117(4):860-868.

89. Eldar S, Bar-Haim Y. Neural plasticity in response to attention training in anxiety. Psychol Med. 2010;40(4):667-677.

90. Levine B, Robertson IH, Clare L, et al. Rehabilitation of executive functioning: an experimental-clinical validation of goal management training. J Int Neuropsychol Soc. 2000;6(3):299-312.

91. Alderman N, Fry RK, Youngson HA. Improvement of self-monitoring skills, reduction of behaviour disturbance and the dysexecutive syndrome: comparison of response cost and a new programme of selfmonitoring training. Neuropsychol Rehabilit. 1995;5:193-221.
92. Ueoka Y, Tomotake M, Tanaka T, et al. Quality of life and cognitive dysfunction in people with schizophrenia. Prog Neuropsychopharmacol Biol Psychiatry. 2011;35(1):53-59.

93. Duncan CC, Barry RJ, Connolly JF, et al. Event-related potentials in clinical research: guidelines for eliciting, recording, and quantifying mismatch negativity, P300, and N400. Clin Neurophysiol. 2009;120(11):1883-1908.

94. Maurage P, Pesenti M, Philippot P, Joassin F, Campanella S. Latent deleterious effects of binge drinking over a short period of time revealed only by electrophysiological measures. J Psychiatry Neurosci. 2009; 34: 111-118.

95. Maurage P, Campanella S, Philippot P, Vermeulen N, Constant E, Luminet O, de Timary P. Electrophysiological correlates of the disrupted processing of anger in alcoholism. Int J Psychophysiol. 2008:70(1):50-62.
Neuropsychiatric Disease and Treatment

\section{Publish your work in this journal}

Neuropsychiatric Disease and Treatment is an international, peerreviewed journal of clinical therapeutics and pharmacology focusing on concise rapid reporting of clinical or pre-clinical studies on a range of neuropsychiatric and neurological disorders. This journa is indexed on PubMed Central, the 'PsycINFO' database and CAS.

\section{Dovepress}

The manuscript management system is completely online and includes a very quick and fair peer-review system, which is all easy to use. Visit http://www.dovepress.com/testimonials.php to read real quotes from published authors. 